\title{
Radiation leakage study for the Valencia applicators
}

\author{
D. Granero ${ }^{\mathrm{a}, *}$, J. Perez-Calatayud ${ }^{\mathrm{b}, \mathrm{c}}$, F. Ballester ${ }^{\mathrm{d}}$, Z. Ouhib ${ }^{\mathrm{e}}$
}

\author{
a Department of Radiation Physics, ERESA, Hospital General Universitario, E-46014 Valencia, Spain \\ ${ }^{\mathrm{b}}$ Radiotherapy Department, Hospital Clinica Benidorm, E-03501 Benidorm, Alicante, Spain \\ ${ }^{\mathrm{C}}$ Physics Section, Radiotherapy Department, Hospital La Fe, E-46009 Valencia, Spain \\ ${ }^{\mathrm{d}}$ Department of Atomic, Molecular and Nuclear Physics, University of Valencia, E-46100 Burjassot, Spain \\ e Lynn Cancer Institute of Boca Raton Regional Hospital, Boca Raton, FL, USA
}

Received 26 February 2011; received in revised form 14 November 2011; accepted 18 November 2011 Available online 9 December 2011

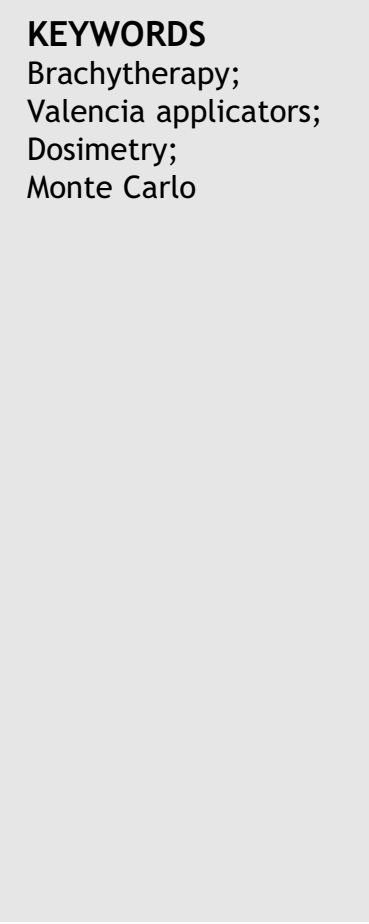

\begin{abstract}
Introduction and purpose: The Valencia applicators which are accessories of the microSelectron-HDR afterloader (Nucletron, Veenendaal, The Netherlands) are designed to treat skin lesions. These cup-shaped applicators are an alternative to superficial/orthovoltage $\mathrm{x}$-ray treatment units. They limit the irradiation to the required area using tungsten-alloy shielding, and are equipped with a tungsten-alloy flattering filter allowing the treatment of skin tumors, the oral cavity, vaginal cuff, etc. The tungsten-alloy thickness to shield radiation is not the same in all parts of the applicators. This fact led us to question whether the leakage radiation differs depending on where it is measured, and whether this may be relevant in some clinical cases. The purpose of this work is to study from the radiation protection point of view the radiation leakage of the Valencia applicators, and provide a solution for current users and for the manufacturer.

Methods and materials: Simulations based on the Monte Carlo (MC) method using the Geant4 code have been realized studying the dose rate distribution in air around the cup of the Valencia applicators. An experimental study with radiochromic film has also been done to measure the dose distribution in the back side of the applicators and to compare it with MC results. Results and conclusions: Radiation leakage of up to $170 \%$ of the prescribed dose has been found at the back surface of these applicators. Although this side is not usually directed to the patient, in some applications such as the treatment of a lesion on the nose, special care must be taken to avoid unexpected and unnecessary irradiation of the eyes. A possible solution could be to add additional shielding to the applicator in order to reduce this leakage or to put some shielding to protect the eyes. Additionally, a new concept design of the Valencia applicators using more shielding material in the applicator backside is proposed.

(c) 2011 Associazione Italiana di Fisica Medica. Published by Elsevier Ltd. All rights reserved.
\end{abstract}

\footnotetext{
* Corresponding author. Department of Radiation Physics, ERESA, Hospital General Universitario, Avda. Tres Cruces 2, E-46014 Valencia, Spain.

E-mail address: dgranero@eresa.com (D. Granero).
}

1120-1797/\$ - see front matter ( $) 2011$ Associazione Italiana di Fisica Medica. Published by Elsevier Ltd. All rights reserved. doi:10.1016/j.ejmp.2011.11.006 


\section{Introduction}

The Valencia applicators [1] are used in brachytherapy to treat shallow skin lesions up to $3 \mathrm{~cm}$ in diameter and $3 \mathrm{~mm}$ deep. There are two types of applicators: $2 \mathrm{~cm}(\mathrm{VH} 2)$ and $3 \mathrm{~cm}(\mathrm{VH} 3)$ in diameters. These applicators are part of the accessories of the remote afterloader microSelectron HDR (Nucletron B.V., Veenendaal, The Netherlands). The Valencia applicators were designed taking as base the Leipzig applicators [2-5] and adding to them a flattening filter to improve dose homogeneity. This filter produces flat dose rate distributions with a small penumbra that allows focusing the radiation to the target while irradiating minimum part of normal tissue. For these applicators, treatments are given usually in 6-7 fractions of 7-6 Gy (prescribed at $3 \mathrm{~mm}$ of depth) to deposit a total dose of approximately $42 \mathrm{~Gy}$.

In the study of Hwang et al. [4] on the Leipzig applicators, a measurement done using radiographic film shows that these applicators have some radiation leakage in its backside, although its magnitude was not given. In the clinical applications, this radiation is not directed towards the patient in the usual skin treatments. However, in certain situations where the lesion is located on the side of the nose (superior and close to the eyes) the backside of the applicators could possibly be directed towards the eyes. For these kind of set up (or similar ones) special caution must be taken to ensure that the dose received by the eyes due to the radiation leakage is kept as low as reasonably achievable.

Since the Valencia applicators are a design modification of the Leipzig applicators, we expect that the same, or larger, leakage will be present in the Valencia applicators. This is due to the presence of the additional filter used to equalize the dose rate distributions laterally that causes longer treatment times and consequently increased leakage dose.

The purpose of this work is to study the magnitude of the radiation leakage in the Valencia applicators by using Monte Carlo (MC) methods and radiochromic film (RF) measurements. An alternative concept design of the Valencia applicators backside adding shielding material is proposed to reduce the radiation leakage.

\section{Methods and materials}

The cup-shaped Valencia applicators are made of a tungsten-alloy to minimize the radiation leakage and to limit the radiation field to the target [1]. In Fig. 1, a schematic view of the applicators is shown.

\section{Radiochromic film measurements}

Experimental dosimetry using EBT radiochromic film (ISP, Wayne, NJ) was performed to quantify the leakage in the backside of the applicator. The experimental set-up is shown in Fig. 1. It consists basically of small RF pieces located at 6 different depths, three films just outside of the backside of the applicator and the other three ones between the PMMA plates with $2 \mathrm{~mm}$ in thickness. The RF pieces have been irradiated to a dose of about 200 cGy. The methodology used for RF dosimetry has been described

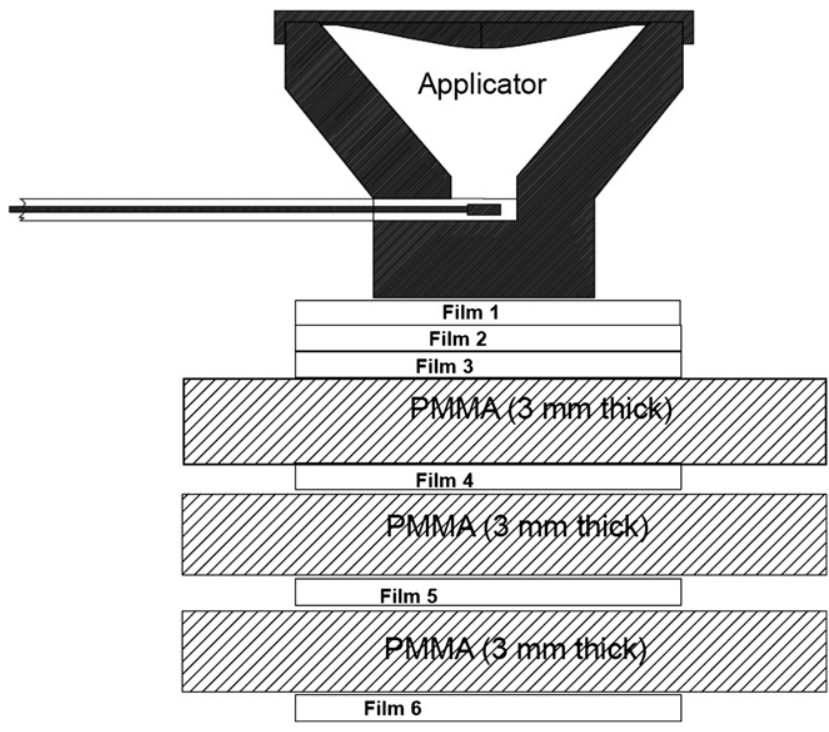

Figure 1 Schematic view of the set-up for measurements with EBT radiochromic film. Distances are in $\mathrm{mm}$. The distances in the figure are not to scale.

elsewhere [1]. The radiochromic films have been irradiated in a Co-60 Phoenix machine to doses ranging from 10 to 600 cGy. The films were read $24 \mathrm{~h}$ after irradiation in a Microtek flat-bed scanner. The analysis of the irradiated films was done using Matlab (MathWorks, Massachusetts)and Verisoft (PTW, Germany) software. We have followed the study of Devic et al. [6] in the process of scanning and readout of the films. Although we have obtained 2D dose distributions in the RF pieces, we are only interested in the maximum value of the leakage in the backside of the applicator. The position of the maximum leakage will be in an axis coincident with the $z$ axis, that is, in the center of the applicator were the shielding material is minimum. To obtain this maximum value, the films have been analyzed in a 2D mode and the maximum leakage in a small ROI has been obtained to compare with MC simulations. The uncertainty has been generated combining those obtained in the calibration of the RF films and the statistical ones obtained in the measurement process of the leakage by means of repeated measurements. The uncertainty in the complete handling and reading process of the RF films has been estimated of about $6 \%(k=1)$.

\section{Monte Carlo estimation of the radiation leakage}

To estimate the Valencia applicator radiation leakage in its backside, MC radiation transport simulations have been performed using the GEANT4 code (version 9.0) [7]. The materials, dimensions of the applicators and the MC methodology (cut-off energy, physics processes used, etc) are the same as described in the study of Granero et al. [1] for the Valencia applicators. Dose rate distributions have been scored in a 3D fashion using a grid of $200 \times 200 \times 200$ cubic cells with $0.5 \mathrm{~mm}$ on a side. In the present study, the applicator has been located on a water cylinder $10 \mathrm{~cm}$ in radius and $10 \mathrm{~cm}$ in height in full contact to its surface. The 
water collision kerma values have been obtained for the treatment zone and air collision kerma for the backside of the applicator as we are interested in the radiation protection of the patient. Thus, we think that collision kerma in air is appropriate to get an estimation of the leakage. Collision kerma in air and water have been scored to estimate dose to speed up the simulations. The linear track length kerma estimator [8] has been used. Up to $5 \times 10^{8}$ photon histories have been simulated obtaining statistical uncertainties $<1 \%$ $(k=1)$ in the kerma results for the points of interest in this study.

An additional MC simulation has been done following the geometry described in Fig. 1 by simulating the PMMA plates and the RF pieces. In this simulation we have estimated absorbed dose in the RF films instead of water collision kerma tracking secondary electrons. The purpose of this additional simulation is to verify that we have not introduced any error in the geometry and materials in the code used. With results of this simulation we have performed a rough comparison with the RF measurements taken into account the contribution of the secondary electrons on films 1,2 and 3 as shown in Fig. 1. We have simulated enough photon histories to get a statistical uncertainty in the dose values less than $5 \%(k=1)$.

\section{New design of the Valencia applicators}

Once verified by RF measurements that the $M C$ estimation of the Valencia applicator leakage are correct within uncertainties, an analytical estimation of the extra shielding needed to reduce the leakage on the applicators backside to a certain level was done. The method uses the fact that the main contribution of the added shielding to the dose rate distributions will be the multiplication of the original dose distribution by the exponential $\exp (-\mu x), \mu$ being the effective attenuation coefficient of the shielding material for the mean energy of the ${ }^{192} \mathrm{Ir}$ spectrum and $x$ the required shielding thickness. By inverting it we will obtain a good approximation to the desired shielding thickness. The material used in the filters is similar to the Valencia applicators. If we suppose and effective attenuation coefficient of about $3.1 \mathrm{~cm}^{-1}$ for this material and ${ }^{192}$ Ir mean energy, $4 \mathrm{~mm}$ thickness of it will reduce the dose to its $30 \%$ value (approximately) without taking into account the additional reduction caused by the inverse square law factor as we are further away from the source.

Finally, an MC study similar to that described in section 2 was done to verify that this analytical estimation of the extra shielding material was correct.
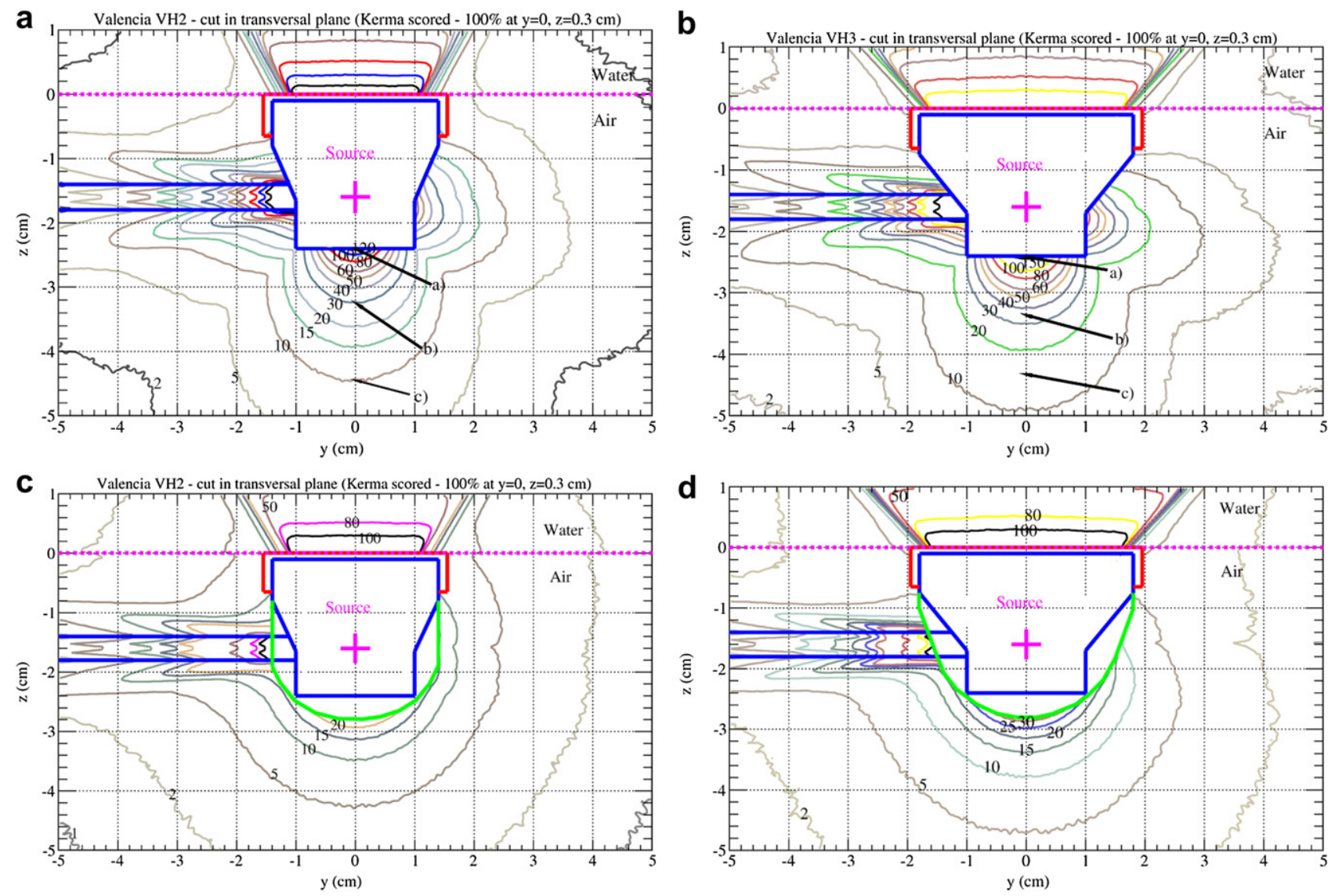

Figure 2 Figures (a) and (b) displays water and air collision kerma in the Valencia VH2 and VH3 applicators respectively, while figures (c) and (d) displays it for the applicators with extra shielding added (green line). Radiation leakage is apparent comparing top to bottom figures. The source centre is indicated by the cross. Dotted lines represent separation between water and air media. The plastic treatment cap has been considered in the simulations. (For interpretation of the references to colour in this figure legend, the reader is referred to the web version of this article.) 


\section{Results and discussion}

\section{Monte Carlo estimation of the radiation leakage}

The results of the $M C$ study of the Valencia applicators are shown in Fig. $2 a$ and $b$. The images correspond to a transversal profile along the applicator axis. The applicators are also schematically shown. Collision kerma has been normalized to its value at the prescription point located at $z=3 \mathrm{~mm}$ and $y=0 \mathrm{~mm}$, i.e. @ $3 \mathrm{~mm}$ depth.

As could be seen in Fig. $2 a$ and $b$, the radiation leakage outside the Valencia applicators are significant. For the VH2 applicator, the values are approximately $130 \%$ at the back surface (point a), 30\% at $1 \mathrm{~cm}$ (point b), and 10\% at $2 \mathrm{~cm}$ (point $\mathrm{c}$ ). The corresponding values for the $\mathrm{VH} 3$ are higher: $170 \%, 35 \%$ and $15 \%$, respectively. These differences are the result of a thicker filter on the $\mathrm{VH} 3$ and similar shielding thickness on the back for both applicators ( $\mathrm{VH} 2$ and $\mathrm{VH} 3$ ). Consequently, treatment times are $32 \%$ longer for the $\mathrm{VH} 3$ applicator than for the $\mathrm{VH} 2$ applicator to deposit the same dose at $3 \mathrm{~mm}$.

\section{Radiochromic film measurements}

Fig. 3 shows RF measurements performed as schematically depicted in Fig. 1 for the $\mathrm{VH} 3$ applicator. The comparison of the $M C$ results with the RF measurement data shows an agreement of about $\pm 10 \%$ at distances greater than $1 \mathrm{~mm}$

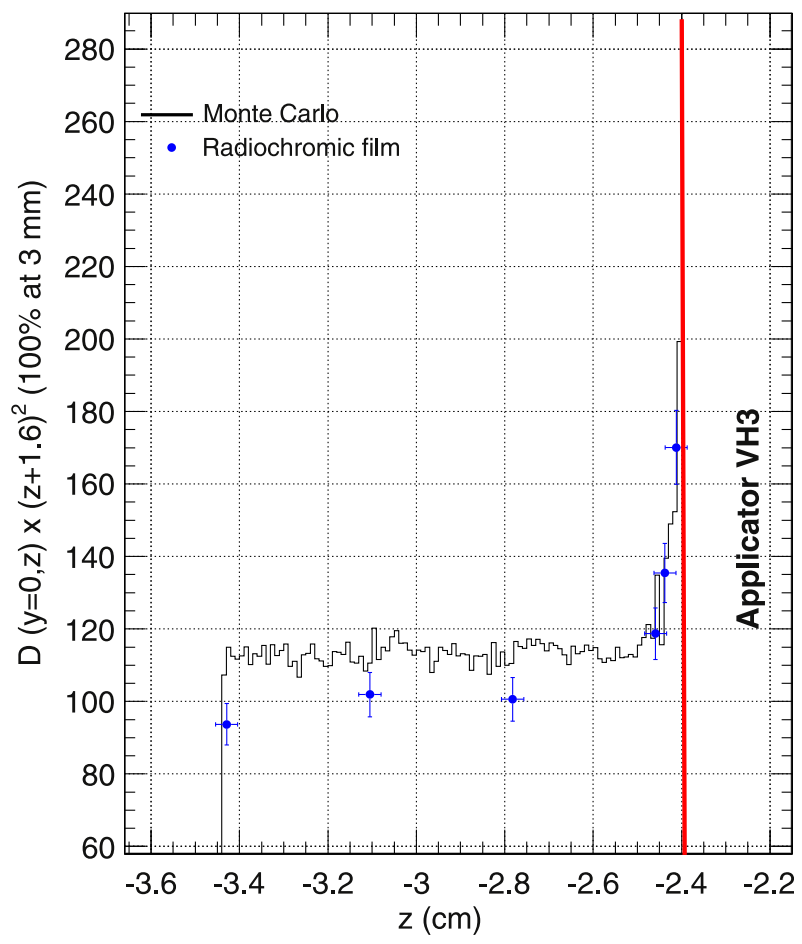

Figure 3 RF measurements along the symmetry axis of the Valencia applicators. Measurements were performed as schematically shown in Fig. 1 for the $\mathrm{VH} 3$ applicator. Points represent RF measurement and solid line $M C$ results. Results are presented $\times r^{2}$ to remove geometrical dependence of the data to clearly show agreement/differences. from the applicator back side. Differences up to $20 \%$ are present at distances less than $1 \mathrm{~mm}$. The causes of these differences are mainly due to the uncertainties in the positioning of the films, about $0.25 \mathrm{~mm}$. These uncertainties produce large dose differences in this region as there is a high dose gradient due to the contribution of secondary electrons just outside the applicator that enhance dose at very short distances.

$\mathrm{RF}$ measurement results show that we have no error in the $M C$ simulation as $M C$ results for the Valencia applicators leakage are in good agreement within uncertainties with the RF measurements taking into account the approximations assumed: the leakage is more significant just in the backside of the applicators. This is due to the presence of secondary electrons generated in the tungsten-alloy. In the treatment zone of the applicator these electrons are eliminated using a plastic cap.

\section{New design of the Valencia applicators}

As explained in section II.3, we have estimated the extra shielding needed to be added in the backside of the $\mathrm{VH} 2$ and $\mathrm{VH} 3$ applicators to reduce the radiation leakage to approximately $10 \%$ @ a distance of $10 \mathrm{~mm}$ from the new backside (green line in Fig. $2 c$ and d). This is equivalent to an additional shielding of $4 \mathrm{~mm}$ (approximately) of tungsten-alloy in the applicator backside. In the current design, the tungsten-alloy part has a material thickness (from the source to the outside geometry) of $6.75 \mathrm{~mm}$. Adding $4 \mathrm{~mm}$ of tungsten-alloy to the current design of the Valencia applicator will yield a total tungsten-alloy material thickness of $10.75 \mathrm{~mm}$. The final new design of the $\mathrm{VH} 2$ and $\mathrm{VH} 3$ applicators backside is displayed by the green lines in Fig. 2c and d.

$M C$ results for these new designs of the $\mathrm{VH} 2$ and $\mathrm{VH} 3$ applicators are displayed in Fig. 2c and d. Comparison with the leakage present in the current $\mathrm{VH} 2$ and $\mathrm{VH} 3$ applicators is easily made by visual inspection of the isokerma lines in Fig. $2 a$ and $b$.

\section{Discussion}

The results of this study show that radiation leakage in the $\mathrm{VH} 2$ and $\mathrm{VH} 3$ applicators must be taken into account in clinical cases. This is important when the back surface of these applicators is oriented towards critical structures such as the eyes (nose set-up). In these particular situations the user should either make every effort to adjust the applicator such that the back surface is oriented away from these structures or by adding some lead as shielding, as this being done during treatment with megavoltage electrons. Moreover, the applicators must not be fixed to the lesion using patient's hands. Let's suppose that this lead will attenuate the radiation in an exponential way. Therefore for the $\mathrm{VH} 2$ applicator, the addition of $2 \mathrm{~mm}$ of lead to the back side of the applicator will reduce the leakage to $40 \%$ of the dose at the prescription point; $4 \mathrm{~mm}$ of lead will reduce the dose to the $20 \%$; and $6 \mathrm{~mm}$ to $10 \%$. For the $\mathrm{VH} 3$ one, the reduction will be approximately of $60 \%, 25 \%$ and $10 \%$ for 2,4 and $6 \mathrm{~mm}$ of lead, respectively. In addition, some plastic or wax material must be fixed to the lead in order to eliminate 
the contribution of secondary electrons at very short distances. As it could be observed in Fig. 3 the contribution of secondary electrons produces an increase of the dose for distances less than $0.5 \mathrm{~mm}$ from the metallic surface of the applicator. Thus it seems appropriate adding $0.5 \mathrm{~mm}$ of some plastic material, as that used in the plastic cap of the applicator, to remove these secondary electrons. Differences between RF measurements and MC results are always within expanded uncertainties $(k=2)$. So taking it into account we could conclude that RF measurement supports $M C$ results.

New prototypes of Valencia applicators incorporating additional shielding material in the back surface have been manufactured by Nucletron. This additional shielding would produce a slight increase in the mass of the original applicator of about $70 \%$ although the weight of the applicator remains low. The size of the applicator will only increase its thickness by $4 \mathrm{~mm}$ on its backside. The selection of this extra shielding has been based on the efficiency of shielding and low weight of the applicator, keeping the applicator integrity, handily and easy of use as in the current version.

\section{Conclusions}

In this study, MC and RF measurements of the radiation leakage were performed for the Valencia skin applicators. Significant leakage, up to $170 \%$ of the prescribed dose has been found just outside the backside of the applicator. Although in most cases radiation leakage are not clinically relevant, because they are not directed to the patient skin, there are some cases where caution must be exercise to not irradiate normal tissue. An example of these situations is the treatment of a lesion in the nose where the back side of the applicator might be directed towards the eyes. In those cases some additional lead shielding could be mounted to the back surface of the applicator to reduce the radiation leakage. The patient should never hold the applicator with his fingers during treatment.

Based on MC results, the proposed new concept design of the Valencia applicator back surface with some additional shielding material to rectify the current issues in the specific situations reported in this paper is being developed by the manufacturer, offering a safe and practical solution for patients and users.

\section{Acknowledgments}

This study was supported in part by Nucletron, B.V. (The Netherlands); Generalitat Valenciana, (Project PROMETEO2008/114); and Ministerio de Ciencia e Innovación, Spain (Project No. FIS2010-17007).

\section{References}

[1] Granero D, Pérez-Calatayud J, Jimeno J, Ballester F, Casal E, Crispín V, et al. Design and evaluation of a HDR skin applicator with flattening filter. Med Phys 2008;35:495-503.

[2] Pérez-Calatayud J, Granero D, Ballester F, Puchades V, Casal E, Soriano A, et al. A dosimetric study of the Leipzig applicators. Int J Rad Oncol Biol Phys 2005;62:579-84.

[3] Niu H, His WC, Chu JCH, Kirk MC. Dosimetric characteristics of the Leipzig surface applicators used in the high dose rate brachy radiotherapy. Med Phys 2004;31:3372-7.

[4] Hwang IM, Lin SY, Lin LC, Chuang KS, Ding HJ. Alternative effective modality of Leipzig applicator with an electron beam for the treatment of superficial malignancies. Nuc Inst Meth A 2003;508:460-6.

[5] Evans MDC, Yassa M, Podgorsak EB, Roman TN, Schreiner LJ, Souhami L. Surface applicators for high dose rate brachytherapy in aids-related Kaposís sarcoma. Int J Radiat Oncol Biol Phys 1997;39:769-74.

[6] Devic S, Seuntjens J, Sham E, Podgorsak EB, Schmidtlein CR, Kirov AS, Soares CG. Precise radiochromic film dosimetry using a flat bed document scanner. Med Phys ;32:2245-53.

[7] Agostinelli S, Allison J, Amako K, Apostolakis J, Araujo H, Arce P, et al. GEANT4 - A simulation Toolkit. Nuc Ins Meth A 2003;506:250-303. See also, http://geant4.web.cern.ch/ geant4. last accessed 14 November 2011.

[8] Williamson JF. Monte Carlo evaluation of kerma at a point for photon transport problems. Med Phys 1987;14:567-76. 